\title{
Superconducting magnetic heterostructured components for electric motor applications
}

\author{
V. Climente-Alarcon, Member, IEEE, N. Mineev, A. Smara, L. Tomkow, B. A. Glowacki
}

\begin{abstract}
Trapped flux magnets made by stacking high temperature superconducting tape portray an easy assembly with already available materials, high mechanical resistance, provided by the substrate and improved thermal stability, which enhances the trapped flux compared to bulks. This allowed reaching the world record of $17.7 \mathrm{~T}$. The presented analysis expands previous work with wide superconducting tapes showing further possible improvements in this kind of devices in order to be used as source of magnetic flux in electrical motors, substituting permanent magnets. The aims are to increase the trapped magnetic flux during magnetization and decrease the leakage flux at the edges of the magnet during the operation of the machine. This is expected to be achieved by either introducing new materials in-between the individual tapes, such as ferromagnetic layers, or modifying the composition of the substrate. The results of simulations using the $\boldsymbol{H}$-formulation as well as experimental measurements are presented.
\end{abstract}

Index Terms - Superconducting magnets, high temperature superconductors, numerical simulation, magnetization processes, magnetic flux leakage.

\section{INTRODUCTION}

$\mathbf{T}$ HE application field of rotating electrical machines can be manifestly expanded by increasing the magnetic flux density levels in the airgap under which they operate. In a first approximation, the power density of the machine escalates linearly with this magnitude [1] and hence, the introduction of superconducting technology may enable high torque, low speed devices, especially suitable for aircraft propulsion [2] and wind generation [3] applications. Nonetheless, despite the advances in material technology and manufacturing methods that happened in recent decades, commercialization of this kind of devices has not happened yet.

The complexity associated with the electromagnetic, thermal and mechanical requirements needed to operate these machines and the corresponding decrease in reliability may be one of the reasons for this outcome. In this sense, stacks consisting of pilled-up superconducting tape, firstly proposed as

This manuscript was submitted for review on September 19, 2019.

This research is financially supported by the European Union's Horizon 2020 research innovation programme under grant agreement No 7231119 (ASuMED consortium) and EPSRC grant EP/P000738/1.

V. Climente-Alarcon, N. Mineev, A. Smara, L. Tomkow and B. A. Glowacki are with the Applied Superconductivity and Cryoscience Group, University of Cambridge, CB3 0FS, Cambridge, UK, (e-mails: vc363@cam.ac.uk, nm693@cam.ac.uk, as2903@cam.ac.uk,1tt27@cam.ac.uk,bag10@cam.ac.uk).

Bartek A. Glowacki is also with The Institute of Power Engineering, ul. Mory 8, 01-330 Warsaw, Poland.

Color versions of one or more of the figures in this paper are available online at http://ieeexplore.ieee.org.

Digital Object Identifier will be inserted here upon acceptance. field source for compact nuclear magnetic resonance equipment [4] could ease some of these demands [3]. Once induced using a normal field, current vortexes circulate in the superconducting layers as long as cooling is provided [5]. In this manner, flux levels one order of magnitude above the ones provided by the state-of-the-art permanent magnets have been achieved in laboratory conditions [6], prompting the question of their applicability in this same role, -flux source- in the rotor of synchronous machines.

However, the electromagnetic conditions inside a rotating electrical motor differ from those laboratory ones for stationary magnets. First, the supercurrents must be induced in the stacks before operation, preferably without adding further elements to the machine. A way of achieving this would be to utilize the stator mounted winding located at the other side of the airgap. Pulse magnetization techniques can be used if its conductors are conventional ones [7]. On the other hand, for stator coils also wound from superconductor material, field cooling magnetization seems the only reasonable choice. In this case, concerning a fully superconducting machine, the flux trapped by this later magnetization approach is limited by the DC capacity of the stator superconducting coils minus a safety margin to avoid quenching triggered by local imperfections [8]. This magnetizing flux is inversely proportional to the reluctance of the full magnetic circuit, which encompasses in a rotating electrical machine at least the stator yoke, the already mentioned airgap, the rotor yoke and the stacks themselves. Classical conventional configurations also feature stator teeth. The reduction of this total reluctance that limits the magnetization level by using tapes featuring a strongly ferromagnetic substrate was considered in [9], with no improvement observed since the bigger permeability also increases the leakage flux.

Another difference between the operation of stationary magnets and the ones used in rotating electrical machines lies in the fact that the discrete distribution of teeth, if present, and stator winding coils modulate the main flux component in the airgap, yielding high frequency harmonics with significant amplitude. That is, stacks mounted on the surface of the rotor would experiment an AC environment. The normal component of this flux produces in the stacks heating [5], which reduces their performance and must be removed by the cooling system, whereas the tangential components alter the macroscopic current vortexes, yielding demagnetization [10].

In order to avoid those problems created by AC fields, the simpler surface mounted stack configuration used for the simulations in [8] and tested in [11], can be modified by adding 
ferromagnetic material around the trapped-flux magnet, that is, burying the magnets inside the rotor yoke. The rotor yoke shields them producing a mostly a DC electromagnetic environment in what is known as an interior mounted magnet configuration. Based on this idea, a novel rotor layout specifically tailored for stacks operating in a fully superconducting synchronous motor was proposed in [12]. However, the rotor yoke's ferromagnetic material reduces the reluctance between the center and the edges of the magnet, increasing again the leakage flux, as seen in [12].

Thus, the aim of this work is to widen the results published in [9] by studying using numerical means the magnetization process and the leakage flux of an interior mounted superconducting stack inside an electrical machine in order to maximize its linked or useful flux. Furthermore, a solution is proposed consisting of modifying the stack's magnetic permeability across its width. Experimentally, this can be done adding ferromagnetic layers between tapes that only cover selected portions (the center of the stack), but in the long term what it is envisioned is modifying the composition of the substrate itself, following some works by manufacturers [13]. Compared to [9], in which the magnet performance was assessed surrounded by air or partially enclosed in ferromagnetic material (in a configuration similar to the one used in surface mounted permanent magnet motors), here the full magnetic circuit of an elementary machine with interior mounted magnets and open ends, as proposed in [12], is evaluated. With this aim, the rest of the paper is organized as follows: Section II summarizes the finite element model used, Section III presents the simulated results on a heterosubstrate stack, compared in Section IV with some experimental measurements obtained by means of a scan probe using in this case ferromagnetic layers. Finally, Section V yields the conclusions.

\section{FINITE ELEMENT MODEL}

The $H$-formulation is applied to obtain the electromagnetic state during the magnetization process in a surface depicting the cross section of a basic segment of an electrical machine [14]:

$$
\frac{\partial \mu \boldsymbol{H}}{\partial t}+\nabla \times \rho(\nabla \times \boldsymbol{H})=0 .
$$

In (1) the state variable to be solved, the magnetic field $\boldsymbol{H}$, is a vector in that same plane, $\boldsymbol{H}=\left[\begin{array}{ll}H_{X} & H_{Y}\end{array}\right]^{T}$ and the derived quantities electric field and current density, $\boldsymbol{E}=\left[E_{z}\right]$ and $\boldsymbol{J}=\left[J_{z}\right]$ respectively, are perpendicular.

In the superconducting regions, the stacks, $\rho$ in (1) is implemented by means of the E-J power law:

$$
\rho=\frac{E_{c}}{J_{c}}\left(\frac{|J|}{J_{c}}\right)^{n-1} .
$$

The stack regions have been modelled as a homogeneous, that is, behaving as an isotropic bulk with the critical current correspondingly scaled down. Since in this work $I_{c}-J_{c}(|\boldsymbol{B}|, T)$ and $n-n(|\boldsymbol{B}|)$, an inaccuracy is expected to be introduced by the computations from the independence of
$J_{c}$ with the angle of incidence of the magnetic flux density $\boldsymbol{B}$. Nevertheless, the ferromagnetic regions surrounding the stacks cause that both the linked and leaked flux are predominantly normal within the stack and thus this minimizes this error to an estimated 5\% variation, according to previous works [9].

The simplified geometry simulated is shown in Fig. 1. Comprises two half poles of a simplified machine having internal mounted stacks with open ends, as in [12]. This is the minimal span that can be simulated using magnetic insulation as a boundary condition around the whole area, since the magnetic flux density during the magnetization process is symmetrical with respect to the center of each pole and thus parallel to these boundaries. Further characteristics of the geometry are shown in Table I.

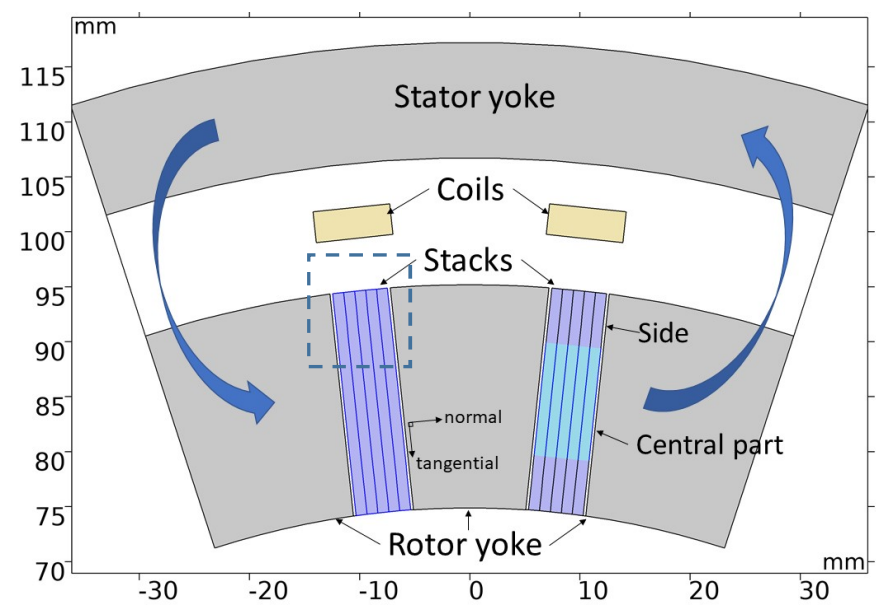

Fig. 1. Geometry simulated comprising two half-poles of an electrical motor. The superconducting stacks embedded in the rotor yoke are depicted in blue. The approximate path of the linked flux is indicated by the wide arrows.

TABLE I

GEOMETRIC AND ELECTRIC CHARACTERISTICS OF THE SEGMENT

\begin{tabular}{lc} 
Stator external diameter & $236 \mathrm{~mm}$ \\
Stator internal diameter & $214 \mathrm{~mm}$ \\
Stator winding distance to center & $101.5 \mathrm{~mm}$ \\
Stator coil cross section & $2.8 \times 7 \mathrm{~mm}$ \\
Airgap length & $12 \mathrm{~mm}$ \\
Stack thickness & $5 \mathrm{~mm}$ \\
Rotor yoke external diameter & $190 \mathrm{~mm}$ \\
Rotor yoke internal diameter & $150 \mathrm{~mm}$ \\
Machine length & $175 \mathrm{~mm}$ \\
Angle of the circular section & $36 \mathrm{deg}$ \\
Coil turns & 6 \\
Temperature of operation & $40 \mathrm{~K}$ \\
Magnetizing current range & $0.2-2.4 \mathrm{kA}$ \\
\hline
\end{tabular}

Regarding the material properties input in the computation, iron-cobalt alloy Vacoflux 50, having a magnetic flux density saturation value of $2.35 \mathrm{~T}$ [15] is used in the stator yoke, whereas standard silicon steel M270-A35 fills the rotor iron regions surrounding the stacks. American Superconductor AMSC (RE)BCO tape is considered for the stacks. This tape features very good characteristics for stacking since it is manufactured in a width of $46 \mathrm{~mm}$ and yet portrays an engineering current density of $4.49 \cdot 10^{8} \mathrm{~A} / \mathrm{m}^{2}(391 \mathrm{~A} / \mathrm{cm}$-width) at $77 \mathrm{~K}$ 
and self-field (Fig. 2). Its thickness is $87 \mu \mathrm{m}$ and the superconducting layer is deposited on a $\mathrm{Ni}-5$ at. $\% \mathrm{~W}$ substrate [6].

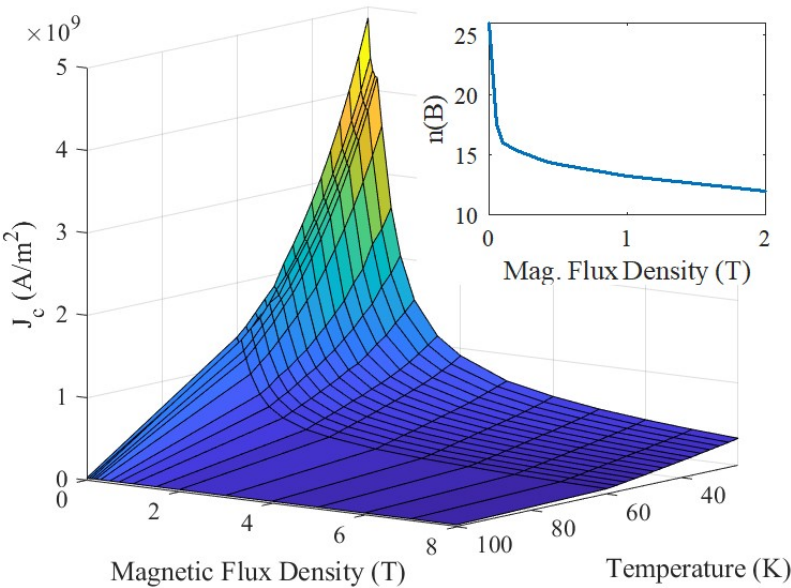

Fig. 2. Superconductor characteristics used in the model.

The time-stepping simulation reproduces a field cooling magnetization of the stacks, performed following the three stages depicted in [8]. Initially, the machine is de-energized. The currents in the two stator coil sides shown in Fig. 1 are then ramped up, being the stacks kept above the critical temperature. In the second one, the rotor is cooled down, in this case to $40 \mathrm{~K}$, and finally, the last part consists of ramping down the currents in the coils. Supercurrents are then induced in the stacks opposing the variation of the decreasing flux.

Seven different levels of magnetizing current have been employed in the simulations, ranging from 0.2 to $2.4 \mathrm{kA}$. Two different stack morphologies have been processed following the results of [9]. The first one with the same relative magnetic permeability in the full stack equal to 10 . This value is obtained experimentally from [6] for the same material in high field conditions. The other configuration consists of a refined design with a value of 1 only at its sides (see Fig. 1), top and bottom, which spans a distance of $5 \mathrm{~mm}$.

The constructive process of such a magnet with different magnetic permeability across its surface would consist either of introducing central ferromagnetic layers between tapes, with inert ones surrounding them at the sides, or there might exist the possibility of growing the superconducting layer on a composite substrate, following [13], with slight ferromagnetic one at its center, such as Ni-5 at.\%W, and having mainly nonmagnetic material at the edges (such as Ni-9.5 at.\%W with a Curie temperature below $25 \mathrm{~K}$ ). In this work this latter option is studied by FE analysis, whereas the first is utilized to obtain some experimental results.

\section{Simulated Results}

The results of the computations carried out with the original and modified stacks are presented in the two points of this section.

\section{A. Flux Leaked through the Edges of the Stack}

Fig. 3 presents the results of integrating the magnetic flux density during the magnetization procedure across the iron yoke surface facing one stack. The upper blue line represents the flux level induced by the coils and follows the classical evolution of the magnetic circuit saturation, whereas the lower red line is the value at the end of the simulation, when all the magnetomotive force comes from the stacks. Clearly this flux, that represents the linked or useful one, decreases as the saturation of the stacks increases. This is caused by the effect of leakage paths. At the magnetizing current of $2.4 \mathrm{kA}$ the percentage of flux lost is $52 \%$. Correspondingly, the bottom dashed curve shows the flux leaked in the whole stack airgap, calculated as the absolute value of the normal flux minus the actual normal flux on the iron yoke. It is obvious that leakage increases with saturation of the stack.

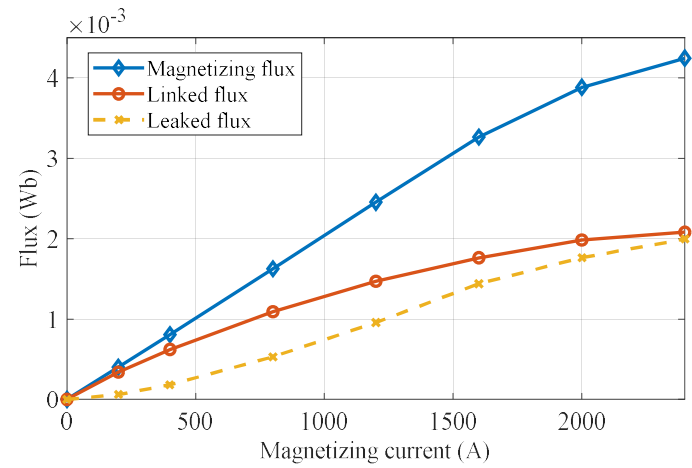

Fig. 3. Increased leakage flux through the stack as its saturation rises.

The cause of such increase can be ascertain examining the magnetic flux and current distribution in the stack area after the magnetization procedure. Fig. 4 a) illustrates the electromagnetic state of the stack's top area (dashed line in Fig. 1) after magnetization with a $0.8 \mathrm{kA}$ current. The color scale represents magnetic flux density in the air and iron region areas, but also in the center of the stack, where no currents are present. The red-blue scale displays instead the supercurrents induced in the stack, in this case outward-bound from the depicted plane (they return at the bottom of the stack not shown here). Smaller arrows represent the circulation of the magnetic flux density, whereas an arrow line has been added along its righthand side edge to show the direction of the flux on that surface. Although supercurrents are present just in the outmost millimeter of the stack, some flux leakage is shown by the inverted arrow at the upper corner. The phenomenon is exacerbated when the current reaches $2.4 \mathrm{kA}$, the saturation of the stack increases and the maximum of current moves further into it (Fig. 4 b)).

It must be noted that an increase of $J_{c}$ in the superconductor would shift the supercurrents back towards the edges of the stack, hence slightly reducing the leakage flux. The conclusion is that in order to operate stacks or bulks of superconducting material between iron parts, the optimal performance is achieved under heavily unsaturated state. 

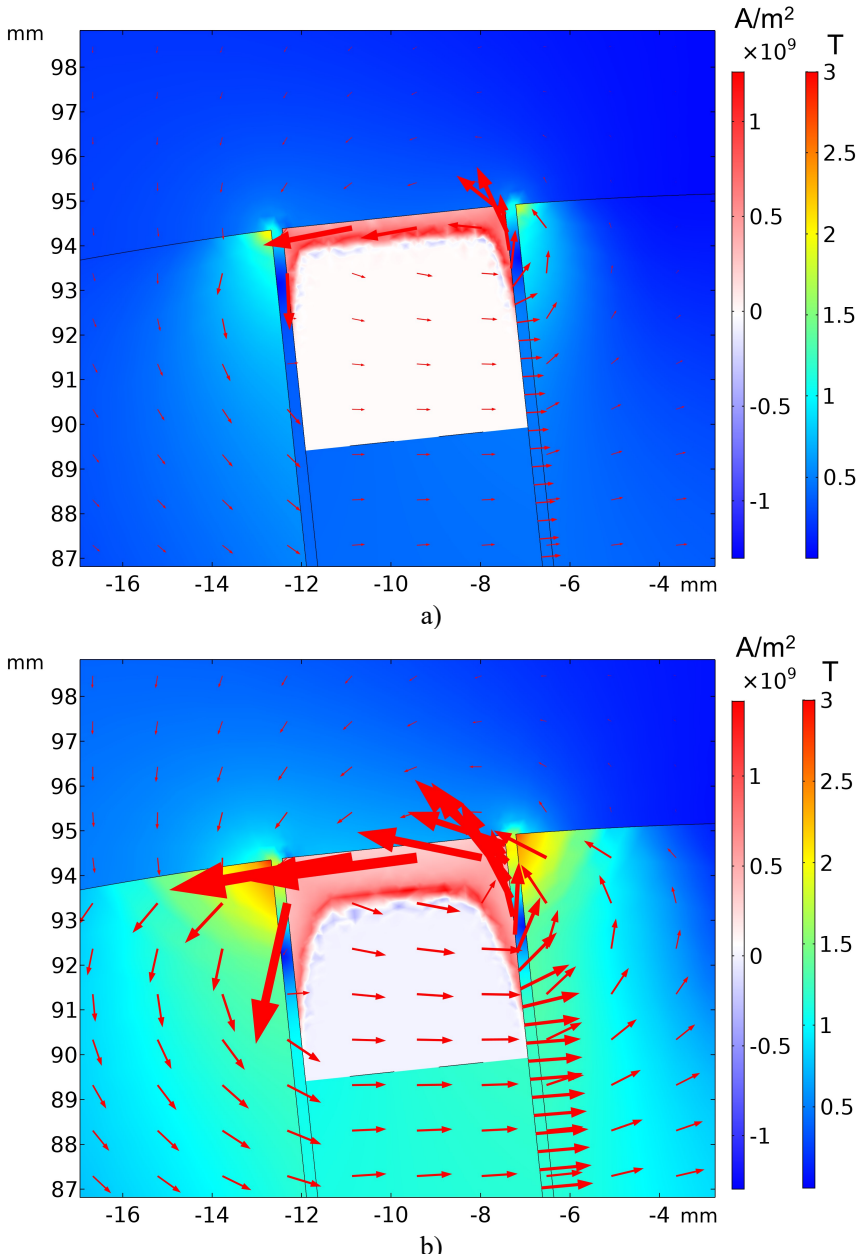

Fig. 4. Distribution of magnetic flux density (color scale) and the current density (red-blue scale) around the top of the left stack after magnetization with currents of $0.8 \mathrm{kA} \mathrm{a}$ ), and $2.4 \mathrm{kA} \mathrm{b}$ ), showing the leaking flux. All arrows depicting the magnetic flux circulation are at the same scale.

\section{B. Improvement of Magnetization by Variable Relative Per- meability of the Substrate across the Stack}

The results of the magnetization procedure for the improved stack with reduced magnetic permeability at its sides are shown in Fig. 5 and compared to the ones in Fig. 3. Contrary to [9], with this hypothetical configuration, the trapped flux can be increased, since, first, it doesn't increase much the reluctance of the full magnetic circuit. This effect is shown in Fig. 5 by a similar magnetizing flux for the same current in both cases. Secondly, the edge leakage, which so much reduced the trapped flux in [9], is limited since both sides of the stacks, where this effect happens, portray a lower magnetic permeability.

Overall, this ideal configuration lowers the magnetizing flux only a $6 \%$, has little effect at lower saturation values of the stack, for magnetizing currents of 0.2 and $0.4 \mathrm{kA}$. However, for higher magnetizing fluxes, it improves the linked flux by two percentage digits, with a $61 \%$ increase for the latest case, $2.4 \mathrm{kA}$.

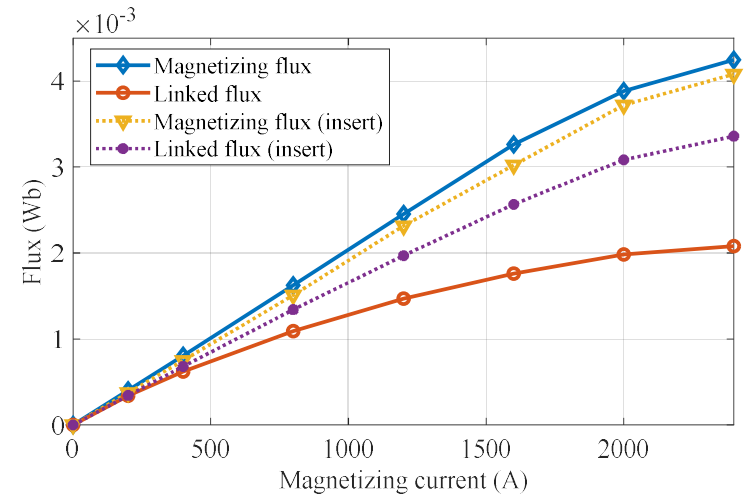

Fig. 5. Comparison of the magnetizing and linked flux between both stack configurations.

\section{EXPERIMENTAL RESULTS}

Experimental measurements of the magnetic flux density $0.75 \mathrm{~mm}$ above the surface of an elemental stack have been taken with different configurations by a Hall probe. The area scanned is limited to $16 \times 16 \mathrm{~mm}$, and hence not the whole trapped field can be depicted. More details about the measurement device can be found in [16].

Three configurations of an AMSC $46 \times 46 \mathrm{~mm}$ two-layer stack have been studied (Fig. 6). The first one consists of just two layers of tape, the second one added a NiFe $100 \mu$ m layer in-between them, covering its full surface, whereas the last one featured an insert of the same material, with a dimension of $19 \times 19 \mathrm{~mm}$, just covering the center of the stack.

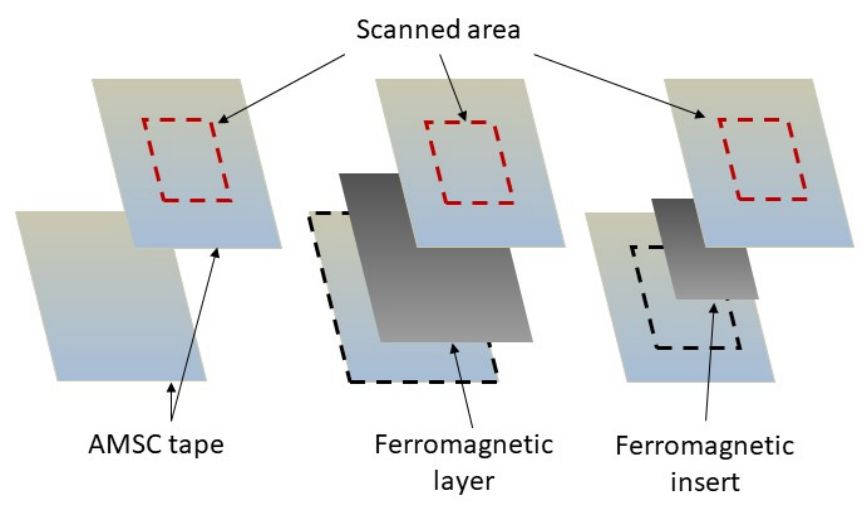

a)

b)

c)

Fig. 6. The three stack configurations experimentally studied. All are composed of two layers of AMSC tape. The scanned area only spans their center.

Zero field cooling has been applied to magnetize all the samples. The maximum magnetizing flux was chosen as 110 $\mathrm{mT}$ in order to avoid fully saturating the first configuration. Fig. 7 portrays the flux profile around the central part of the stack for the two stacks with ferromagnetic material between the tapes. A clear improvement in the maximum trapped magnetic flux density is shown for the case with the insert. The average values measured in the area under the scan were 34.2 $\mathrm{mT}$ for the case without ferromagnetic material, $48.6 \mathrm{mT}$ for the full ferromagnetic layer and $54.6 \mathrm{mT}$ in the latter case, fea- 
turing just the insert. This constitutes an increase of trapped flux of $12 \%$ on the measured surface.

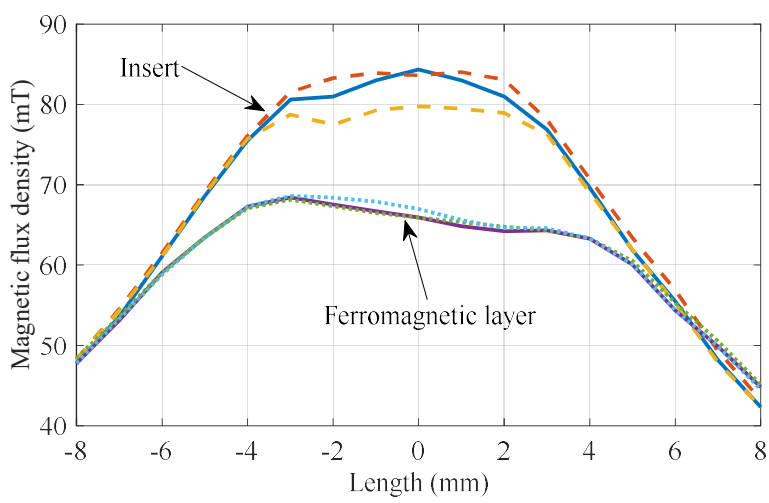

Fig. 7. Magnetic flux density profile around the central part of a two-layer AMSC stack for the two cases featuring ferromagnetic material in-between the tapes. Dashed lines indicate the profile at $\pm 1 \mathrm{~mm}$ from the central one.

\section{CONCLUSIONS}

The state-of-the-art trapped flux magnets cannot work in the airgap of a rotating electrical machine due to losses and demagnetization caused by the harmonics present in that environment. Interior mounted solutions, also used in conventional machines, suffer increased leakage with saturation as the surrounding ferromagnetic material provides low reluctance paths along the surface of the stack. Arrangements with heterogenous layers, having a different magnetic permeability between its sides and center, can solve this problem. In the long term, this variation of permeability could be included during the manufacturing process by modifying the substrate itself.

\section{REFERENCES}

[1] V. Climente-Alarcon, A. Patel, A. Baskys, B. A. Glowacki, "Design considerations for electric motors using stacks of high temperature superconducting tape as permanent magnets," IOP Conf. Ser.: Mater. Sci. Eng., vol. 502, 2019, Art. no. 012182, (doi: 10.1088/1757$899 \mathrm{X} / 502 / 1 / 012182)$

[2] K. S. Haran et al., "High power density superconducting rotating machines-development status and technology roadmap," Supercond. Sci. Technol., vol. 30, 2017, Art. no. 123002, (doi: 10.1088/13616668/aa833e)

[3] X. Song, C. Buhrer, P. Brutsaert, et al., "Designing and basic experimental validation of the world's first MW-class direct-drive superconducting wind turbine generator," IEEE Trans. Energy Convers., vol. 34, no. 4, pp. 2218-2225, Dec. 2019, doi: 10.1109/TEC.2019.2927307.

[4] S. Hahn, J. Voccio, S. Bermond, D.-K. Park, J. Bascuñán, S.-B. Kim, T. Masaru, Y. Iwasa, "Field Performance of an Optimized Stack of YBCOSquare "Annuli" for a Compact NMR Magnet," IEEE Trans. Appl. Supercond., vol. 21, no. 3, Jun. 2011, pp. 1632-1635, (doi: 10.1109/TASC.2010.2103920).

[5] Y. Iwasa, "Case Studies in Superconducting Magnets, Design and Operational Issues," Second edition, Springer, 2009.

[6] A. Patel, A. Baskys, T. Mitchell-Williams, A. McCaul, W. Coniglio, J. Hänisch, M. Lao, B. A. Glowacki, "A trapped field of $17.7 \mathrm{~T}$ in a stack of high temperature superconducting tape," Supercond. Sci. Technol, vol. 31, 2018, Art no. 09LT01, (doi: 10.1088/1361-6668/aad34c).

[7] Shengnan Zou, "Magnetization of High Temperature Superconducting Trapped-Field Magnets," PhD Thesis, KIT Scientific Publishing, 2017, [on-line] Available: https://publikationen.bibliothek.kit.edu/1000073152
[8] V. Climente-Alarcon, A. Patel, A. Baskys, B. A. Glowacki, "Computation of Superconducting Stacks Magnetization in an Electrical Machine," IEEE Trans. Appl. Supercond., Vol 29, no. 8, 5204506, Dec. 2019 (doi: 10.1109/TASC.2019.2923537).

[9] A. Baskys, A. Patel, V. Climente-Alarcon, B. A. Glowacki, "Remanent Magnetic Flux Distribution in Superconducting-Ferromagnetic Layered Heterostructures", Journal of Superconductivity and Novel Magnetism, 2019, (doi: 10.1007/s10948-019-5022-7).

[10] M. Baghdadi, H. S. Ruiz, J. F. Fagnard, M. Zhang, W. Wang, T. A. Coombs, "Investigation of Demagnetization in HTS Stacked Tapes Implemented in Electric Machines as a Result of Crossed Magnetic Field," IEEE Trans. Appl. Supercond., vol. 25, no. 3, Jun. 2015, Art. no. 6602404, (doi: 10.1109/TASC.2014.2372873).

[11] A. Smara, N. Mineev, V. Climente-Alarcon, A. Patel, A. Baskys, B. A. Glowacki, T. Reis, "Experimental Assessment of Rotor Superconducting Stack Demagnetization in LN2 Environment," Superconductor Science and Technology, vol. 32, no. 8, 085009, 2019, (doi: 10.1088/13616668/ab20bf).

[12] V. Climente-Alarcon, A. Smara, A. Patel, B. A. Glowacki, A. Baskys, T. Reis, "Magnetization and Losses for an Improved Architecture of Trapped-Flux Superconducting Rotor," AIAA Journal of Propulsion and Power, (accepted for publication, doi: 10.2514/1.B37709).

[13] J. H. Claassen, C. L. H. Thieme, "Magnetic properties of Ni-based substrates for HTS tape," Supercond. Sci. Technol., vol. 21, 2008 Art. no. 10500, (doi: 10.1088/0953-2048/21/10/105003).

[14] Z. Hong, A. M. Campbell, T. A. Coombs, "Numerical solution of critical state in superconductivity by finite element software," Supercond. Sci. Technol., vol. 19, no. 12, 2006, pp. 1246-1252, (doi: 10.1088/09532048/19/12/004)

[15] F. Fohr, N. Volbers, J. Gerster, "High strength iron-cobalt materials for magnetic bearings," in Proc. ISMB 2014, Linz, Austria.

[16] L. Tomkow, A. Smara, V. Climente-Alarcon, B. A. Glowacki, "Distribution of Trapped Magnetic Flux in Superconducting Stacks Magnetised by Angled Field," J. Supercond. Nov. Magn. (2019) doi:10.1007/s10948-019-05375-3 